\title{
Does the compatibility effect in the race Implicit Association Test reflect familiarity or affect?
}

\author{
SACHIKO KINOSHITA and MARIE PEEK-O'LEARY \\ Macquarie University, Sydney, New South Wales, Australia
}

\begin{abstract}
In the Implicit Association Test (IAT; Greenwald, McGhee, \& Schwartz, 1998) involving race classification (white vs. black), an apparent compatibility effect is found between the "pleasant" attribute and the "white" category. This race IAT effect has been interpreted in terms of "implicit prejudice"that is, more positive evaluation of whites than of blacks that is not open to consciousness. We suggested instead that the race IAT effect is better interpreted in terms of the salience asymmetry account proposed by Rothermund and Wentura (2004), whereby greater familiarity with the white category makes it more salient. Evidence that has been presented against the familiarity interpretation is considered, and alternative interpretations of findings related to the race IAT effect are discussed.
\end{abstract}

In the Implicit Association Test (IAT; Greenwald, McGhee, \& Schwartz, 1998), subjects make speeded classification of two kinds of stimuli: targets and attributes. The target categories comprise the concepts of interestfor example, black Americans versus white Americans or flowers versus insects. In target classification, subjects are asked to classify exemplar stimuli (e.g., first names, such as Meredith, Alan, Ebony, or Jamal, or photographs of white Americans and black Americans) into the target categories. The attributes involve an evaluative dimension, such as pleasant versus unpleasant, and subjects classify stimuli (e.g., words such as love, peace, abuse, or disaster) into the attribute categories. The critical phase of the task involves the blocks in which the target and attribute classification trials are interleaved. In this phase, the assignment of responses to the target and attribute categories may be arranged so that they are "compatible"; for example, the flower and pleasant categories are assigned the right-hand key and the insect and unpleasant categories are assigned the left-hand key. Alternatively, the response assignment may be "incompatible" (where the flower and unpleasant categories are assigned the same key and the insect and pleasant categories are assigned the other key). The IAT effect refers to the faster (as well as more accurate) responses in the compatible block than in the incompatible block, typically on the order of 100-200 msec, and is assumed to reflect the more positive evaluation of one target category (e.g., flowers) than the other (e.g., insects).

The IAT has rapidly gained a large following among social cognition researchers as a measure of "implicit" attitude. Indeed, in the IAT, subjects are not explicitly re-

Correspondence concerning this article may be addressed to $\mathrm{S}$. Kinoshita, Macquarie Centre for Cognitive Science and Department of Psychology, Macquarie University, Sydney, NSW, Australia 2109 (e-mail: sachiko@maccs.mq.edu.au). quired to evaluate their preference toward the target categories (e.g., white vs. black Americans); instead, the IAT effect emerges indirectly. Also, unlike the traditional selfreport measures which are open to self-presentational forces like social desirability and political correctness, the IAT affords little opportunity for deliberate, intentional strategies to influence performance (see Kim, 2003, for a demonstration that instructing subjects to pretend that they liked one category more than the other did not change the size or direction of the IAT effect). These features of the IAT support the claim that it is an "implicit" measure. However, it is not clear whether the IAT actually taps valence (positive or negative evaluation). In this article, we review the evidence that has been used to argue the case that the IAT effect observed with race is based on affective valence. To anticipate our conclusion, we will argue that the case is weak and suggest that the race IAT effect is instead based on relative familiarity of one category (e.g., white) over the other (e.g., black).

Before turning to the review, we raise a theoretical issue that motivated our analysis, one with broad implications for research on implicit attitude in general. It concerns the definition of implicit attitude. The term implicit has many senses, and social psychologists have generally focused on "lack of awareness" as the relevant feature. Greenwald and Banaji (1995) defined implicit attitudes as "introspectively undefined (or inaccurately identified) traces of past experience that mediate favorable or unfavorable feeling, thought, or action toward social objects" (p. 8). Similarly, Wilson, Lindsey, and Schooler (2000) defined implicit attitudes as "evaluations that (a) have unknown origin (i.e., people are unaware of the basis of their evaluation); (b) are activated automatically; and (c) influence implicit responses, namely, uncontrollable responses and ones that people do not view as an expression of their attitude and thus do not attempt to control" (p. 104). 
Implicit attitude is thus defined as a theoretical construct. In their review of implicit attitude research, Fazio and Olson (2003) pointed out problems with this definition, and argued that the term "implicit" should be used to refer to measures instead. This is an important point and one that has been made in the context of implicit memory by researchers such as Jacoby (1996) and Whittlesea and Price (2001). Specifically, Jacoby pointed out that defining "implicit" to refer to constructs is based on what he called a "task-pure" assumption, which assumes that an implicit memory measure taps implicit memory, a process or a construct, and an explicit memory measure taps explicit memory. More colorfully, Whittlesea and Price commented that defining "implicit" as "introspectively unidentified" is made from a "consciousnesschauvinist" (p. 243) position, that it is a "distinction made from the point of the view of the consciousness of the subject, the consumer of memory's products, rather than from the point of view of the mechanisms of memory that produce those products" (p. 243). Instead of "reifying the implicit/explicit dichotomy as a principle of memory itself," Whittlesea and Price advocated that the distinction be used simply as a "handy description of subject's task" (p. 244).

Defined this way, implicit measures of attitudes are simply tasks that are designed to tap subjects' attitudes without making explicit reference to evaluations (like the IAT). Using the term implicit in this way (to refer to task orientation) means giving up the task-pure assumption: An implicit measure of attitude does not necessarily solely index implicit attitudes, nor does an explicit measure of attitude solely tap explicit attitudes (in fact, the latter point is readily appreciated by researchers who recognize that the self-report measures of attitudes are open to extraneous influences like self-presentational forces). Instead, an implicit attitude measure must "earn its keep"- the mechanism by which the task outcome is produced must be understood if the measure is to be of any use.

As pointed out by Fazio and Olson (2003), however, research concerning the IAT has been "surprisingly atheoretical" (p. 301). Although there are studies reporting high consistency among different measures of racism, including the race IAT (e.g., Cunningham, Preacher, \& Banaji, 2001; but see also Olson \& Fazio, 2003), these studies do not provide evidence that the race IAT effect reflects valence. Also, the few studies that have investigated mechanisms (reviewed below) do not suggest that all IAT effects can be explained by the same mechanism.

The rest of the article is organized as follows. We first present a brief review of mechanisms that have been proposed to account for the IAT effect (the Mechanisms Underlying the IAT section). We should note that the purpose of the review is not to present a comprehensive evaluation of all of the accounts proposed to date, ${ }^{1}$ but instead, our focus is on two classes of mechanisms-one based on the idea that the IAT effect is based on affective valence and the other based on salience asymmetry. We then suggest that the race IAT effect is best explained in terms of the latter and will focus our discussion on the role of familiarity as a factor underlying salience asymmetry in the race IAT effect. To this end, we will review studies that claim to have ruled out familiarity as a basis of the race IAT effect and will argue that these studies are in fact consistent with the view that familiarity is a causal factor underlying the race IAT (the Evidence Against Familiarity as the Basis of the Race IAT section). Finally, we reinterpret some of the findings obtained with the race IAT in light of the alternative interpretation (the Alternative Interpretation of the Race IAT Effects section).

\section{MECHANISMS UNDERLYING THE IAT}

The originators of the IAT (Greenwald et al., 1998) believe that the basis of the IAT effect is conceptual association between the target (e.g., flower) and the attribute (e.g., pleasant) categories. The spreading activation account proposed by Greenwald et al. (1998), the taskswitching account proposed by Mierke and Klauer (2001; Klauer \& Mierke, 2004), and the stimulus-response compatibility account proposed by De Houwer (2001) share this assumption, differing primarily in the specific mechanism by which responding is made easier when the same response is assigned to the associated categories than when different responses are assigned to them.

In the case of the spreading activation account, the mechanism is priming within an affective network structure: The target category (e.g., flower) is assumed to receive greater priming from the associated attribute category (e.g., pleasant) and therefore result in faster responses. The task-switching account (Mierke \& Klauer, 2001) suggests that the shared valence between target (e.g., flower) and attribute (e.g., pleasant) categories allows the two classification tasks to be reduced to a single task along the valence dimension in the compatible condition. In contrast, in the incompatible condition, this is not possible and hence the two classification task sets must be maintained, resulting in performance cost due to the requirement to switch between tasks (e.g., Meiran, 2000; Rogers \& Monsell, 1995). De Houwer's (2001) account suggests that the IAT effect is structurally similar to stimulus-response compatibility effects in which responses are faster and more accurate when pressing a left key in response to a stimulus presented on the left side of a screen (and a right key in response to a stimulus presented on the right side of the screen) than when response assignment is reversed. The IAT effects are explained as reflecting a similar effect of assigning the same, as opposed to different, responses to associated target and attribute categories.

It is noted that these accounts are generally not fully developed and little direct evidence for the specific mechanisms has been provided. ${ }^{2}$ One exception to this is the task-switching account. Using flowers and insects as the target concept categories, Mierke and Klauer (2001) 
measured the task-switching cost in the combined task phase of the IAT by comparing the nature of the preceding trial. In support of the task-switching account, they found that response times (RTs) were slower when the preceding task was different (e.g., attribute decision preceded by target classification or vice versa) than when it was the same (e.g., attribute decision preceded by attribute decision or target classification preceded by target classification), and that this task-switching cost was greater in the incompatible IAT block than in the compatible IAT block. (Note, however, that this does not rule out other mechanisms, such as stimulus-response compatibility.)

More direct evidence that task switching involves the evaluative dimension was reported by Klauer and Mierke (2004). They hypothesized that in the incompatible IAT block but not in the compatible IAT block, the attributeevaluation task set must be suppressed in order to respond correctly on the flower-insect classification task. Suppressing the task set must make it more difficult to reactivate it, when it is necessary. Thus, when subjects are asked to evaluate flowers and insects as positive or negative, this should be more difficult following an incompatible IAT block than following a compatible IAT block. The results supported this prediction. In addition, this carryover effect was specific to the attribute-evaluation task: Performance on an unrelated color-naming task was unaffected by the compatibility of the preceding IAT block. One point to note about the Klauer and Mierke study, however, is that they used only the flower-insect IAT. Explicit liking measures (e.g., feeling thermometer, semantic differential) are higher for flowers than for insects, but such contrast in explicit liking is not always observed with race categories (Greenwald et al., 1998; Nosek, Banaji, \& Greenwald, 2002). Thus, it is not known whether the finding of a carryover effect that is specific to the attribute dimension would generalize to other types of IAT.

We now turn to the second class of mechanism, which explains the IAT effect in terms of a factor other than affective valence. Here, we focus on Rothermund and Wentura's $(2001,2004)$ salience asymmetry account, since it provides the best-articulated mechanism.

\section{Salience Asymmetry Account}

The main tenet of this account is that the IAT effect is driven by asymmetries in salience between the contrasting categories. Specifically, Rothermund and Wentura $(2001,2004)$ suggested that in the binary classification tasks used in the IAT (e.g., pleasant vs. unpleasant, young vs. old), subjects may simplify the task by focusing on only one of the contrasting categories, effectively turning the task into a detection task. The category that forms the focus would be the more salient of the categories, being the "figure" category (the other, nonsalient, category being the "ground" category). In a combined IAT block, an effect of compatibility of salience asymmetry emerges such that performance would be facilitated if the same response is assigned to the figure category in target classification and the figure category in attribute classification, relative to when different responses to the two figure categories are required. Rothermund and Wentura (2001) provided direct support for this account by experimentally manipulating the focus category using a "go/no-go" instruction. Specifically, they instructed subjects to respond to only one category in the individual target and attribute-classification blocks (e.g., "respond if the item is a young [old] person's name and make no response otherwise"). The results showed that any combination of the target and attribute categories (i.e., "young-pleasant," "old-pleasant," "young-unpleasant," "old-unpleasant") can be made compatible in an IAT as a function of which of the contrasting categories were made the focus by the go/no-go instruction.

Rothermund and Wentura (2001) noted that in the absence of experimental manipulation of salience, salience asymmetry may arise naturally from a number of sources, including linguistic markedness. It is well known that many dimensions used in natural languages have marked and unmarked ends. For example, in the height dimension, "tall" is unmarked because the question "how tall is he?" does not imply that the person is tall, but "short" is marked because "how short is he?" does imply that the person is short (e.g., Lyons, 1977). Thus linguistically, in the pleasant-unpleasant classification, "pleasant" is unmarked and "unpleasant" is marked. Other sources of salience asymmetry are valence and familiarity. Rothermund and Wentura (2001) noted that in a visual search task, detection is faster for a negative, threatening stimulus (e.g., an angry face) among a background of positive stimuli (e.g., a crowd of happy faces) than for a positive stimulus among a background of negative stimuli (e.g., Hansen \& Hansen, 1988; Ohman, Lundqvist, \& Esteves, 2001). Similarly, asymmetries along the dimension of familiarity have been demonstrated via the phenomenon of novel popout (e.g., Strayer \& Johnston, 2000; Wang, Cavanagh, \& Green, 1994). In visual search tasks, an unfamiliar target among familiar distractors pops out, and hence search time is unrelated to set size (the number of distractors), whereas when searching for a familiar target among unfamiliar distractors, search time increases linearly with set size.

Rothermund and Wentura $(2001,2004)$ assumed that these popout phenomena reflect automatic capture of attention and took them to suggest that the negative and the novel capture attention automatically and hence should be considered the figure. We note that the popout phenomena have not always been interpreted in terms of attentional capture (see, e.g., Tipples, Young, Quinlan, Broks, \& Ellis, 2002). Others have suggested that the asymmetric popout phenomena reflect faster rejection of positive and familiar distractors (e.g., Strayer \& Johnston, 2000; Wang et al., 1994), indicating more fluent processing of the familiar and the positive. Furthermore, in general (e.g., in the linguistics and linguistic development literature; see E. V. Clark, 1973; H. H. Clark, 1973), 
it is the linguistically unmarked, the positive, and the familiar that are generally considered to be the figure. Since nothing in the evidence reported by Rothermund and Wentura $(2001,2004)$ contradicts this position, we suggest that the more fluently processed category - that is, the familiar, the positive, and the linguistically unmarkedshould be considered the figure.

\section{Summary and Evaluation}

We described four accounts of IAT effect: spreading activation, task switching, stimulus-response compatibility, and salience asymmetry. One issue not directly addressed by the proponents of these accounts, with the exception of Rothermund and Wentura (2004), is whether all IATs are driven by the same mechanism. In looking at the target categories that have been used in previous IAT studies, there seem to be two classes: In some IATs, the dimension underlying target classification is a priori correlated with the reference attribute dimension; in the other IATs, there is no obvious correlation between the two dimensions. Rothermund and Wentura (2004) suggested that these two classes of IATs are driven by different mechanisms, and we concur. ${ }^{3}$.

The flower-insect IAT is a classic example of the first class of IAT. The category "flower" and the flower exemplars (e.g., rose, tulip) are rated as more positive than the category "insect" and the insect exemplars (e.g., wasp, cockroach) (Greenwald et al., 1998). Thus, as suggested by the task-switching account, in the compatible IAT block, the two classification tasks can be reduced to a single one of detecting the evaluative feature. In the incompatible IAT block, in contrast, the evaluative attribute of the target category exemplars must be actively ignored, as evidenced by Klauer and Mierke's (2004) finding that subjects were substantially slower at evaluating the valence of flowers and insects following an incompatible IAT block than following a compatible IAT block.

Another example of this first type of IAT involves gender stereotyping. Nosek et al. (2002) found the category "male" to be more compatible with the category "science" (with exemplars like physics, engineering) and the category "female" with "liberal arts" (with exemplars like arts, humanities) than the reversed combination. As pointed out by these authors, there is "strong and historically skewed gender distribution in science" (p. 108), and hence the IAT effect observed is likely to be based on such correlation. Consistent with this interpretation of gender IAT, Steffens and Plewe (2001) reported that the nature of the pleasant and unpleasant attribute words - specifically, whether the positive and negative words were female associated (e.g., emphatic, bitchy) or male associated (e.g., independent, brutal) — had an effect on the size of the gender IAT effect. Similarly, the fact that the IAT involving the 2000 presidential candidates as target categories (and using the first and last names and the photographs of the candidates as category exemplars) correlated with the subjects' voting intentions (Nosek et al., 2002) is not surprising, given the assump- tion that in this IAT, the target category dimension (presidential candidates) would be correlated with the attribute dimension.

A priori correlation between the target and attribute dimensions also explains the different patterns of IAT effect found with different subject groups. For example, it has been shown that IAT discriminates between spider and snake phobics (Teachman, Gregg, \& Woody, 2001). When classifying photographs into spiders and snakes, and words (e.g., lethal, secure) into various evaluative categories (e.g., "danger/safety," "disgusting/appealing," "afraid/unafraid," "bad/good"), the direction of the IAT effect was reversed for the two subject groups (with the spider phobic group showing compatibility between spiders and danger, etc., and vice versa). It has also been reported that psychopathic murderers showed less compatibility between the categories "pleasant" and "peaceful" (vs. "violent") than did murderers low in psychopathy (Gray, MacCulloch, Smith, Morris, \& Snowden, 2003). These findings are consistent with the view that the dimensions can be reduced to a single one in the compatible IAT block because the dimensions underlying the target category classification and the reference attribute classification are correlated in the subject group.

In contrast to these IATs, in other IATs there does not seem to be an a priori conceptual correlation between the target and attribute dimensions. For example, Brendl, Markman, and Messner (2001) found that when "insects" and "nonwords" were target categories, the former was found to be compatible with "pleasant," and the latter with "unpleasant." The direction of this IAT effect contrasted with the ratings of goodness of insects and nonwords, which showed significantly lower ratings to insects than to nonwords. De Houwer's (2001) finding that the size of the IAT effect in the British-foreign IAT (with British subjects) did not differ for liked versus disliked British/foreign exemplars is also at odds with the view that the British-foreign dimension is correlated with the attribute (positive-negative) dimension. Perhaps the most problematic finding for the view that the IAT effect is based on a priori correlation between dimensions is that the direction of the IAT effect was reversed by manipulating which of the contrasting categories is to be the focus category, using Rothermund and Wentura's (2004) " go/no-go" instruction.

This second class of IAT seems best explained in terms of salience asymmetry. Specifically, dimensions of two classification tasks in this type of IAT are not correlated, and thus the two classification tasks cannot be reduced to a single conceptual dimension. Nevertheless, there is a compatible and incompatible combination based on salience asymmetry because the two ends of the dimensions (e.g., pleasant vs. unpleasant, insects vs. nonwords) are not equally salient. This notion of salience asymmetry has precedence in the stimulus-response compatibility literature. Cho and Proctor (2003) reviewed stimulus-response compatibility effects in speededresponse tasks and pointed out that there are compatibil- 
ity effects even for orthogonal spatial dimensions like top/bottom and right/left based on salience asymmetry: Performance is facilitated in conditions in which the two salient ends (e.g., top and right) share one response and the two nonsalient ends (e.g., bottom and left) share the other response.

It is worth emphasizing that salience asymmetry based on orthogonal dimensions such as familiarity and linguistic markedness operates at the level of categories: The process of carrying out two classification tasks is facilitated by having a shared response to the figure categories. From this perspective, De Houwer's (2001) finding of equal IAT effects for liked and disliked British and foreign exemplars is entirely consistent with the salience asymmetry account. That is, "British" and "foreign" categories vary in familiarity (with the former category being more familiar to British subjects), and the pleasantunpleasant classification varies in linguistic markedness (as well as valence), producing the apparent pro-British bias in the IAT due to greater salience of the "British" and "pleasant" categories. Because this IAT effect was not based on a common evaluative dimension (i.e., it was not produced by more positive evaluation of the "British" category), the liking of the individual exemplars had no impact on the size of the IAT effect.

These considerations and other facts suggest that the race IAT belongs to this second class of IATs, which are not based on a priori correlation between the target and attribute dimensions but instead on salience asymmetry, and that difference in familiarity of the contrasting race categories is the source of salience asymmetry. A large prowhite bias is found with the white-black IAT, in contradiction to the explicit measure of preference (Nosek et al., 2002). Subjects are often surprised when told that the large prowhite bias shown by their own IAT data indicates "implicit racism" (Monteith, Voils, \& AshburnNardo, 2001). It has also been reported that the percentage of subjects classified as prowhite based on the IAT (70\%-90\% of whites) is higher than the estimate based on other implicit measures of racism $(50 \%-60 \%$; Olson $\&$ Fazio, 2003). These facts are suggestive of the possibility that at least some part of the prowhite IAT effect reflects salience asymmetry, rather than more positive evaluation of whites than blacks.

\section{EVIDENCE AGAINST FAMILIARITY AS THE BASIS OF THE RACE IAT}

We have suggested above that the race IAT effect is better explained in terms of salience asymmetry, rather than a priori correlation between dimensions underlying the two classification tasks. In particular, we believe that greater familiarity with one race group (e.g., white over black; in-group over out-group) is responsible for the prowhite bias and the in-group bias (e.g., pro-Korean bias shown by Korean subjects or pro-Japanese bias shown by Japanese subjects). Against this possibility, some studies claim to have ruled out familiarity as being responsible for the race IAT effect, and the originators of the IAT reaffirmed this position recently (Dasgupta, Greenwald, \& Banaji, 2003). We will now review findings presented in these studies, which include experimental, statistical, brain imaging, neuropsychological, and behavioral data. We conclude with a discussion of the mere exposure effect, a phenomenon often used to argue for the view that familiarity directly increases affect.

\section{Experimental and Statistical Evidence}

In a study often cited as ruling out familiarity as the basis of the race IAT effect, Dasgupta, McGhee, Greenwald, and Banaji (2000) reported two pieces of evidence. We discuss each in turn.

The first piece of evidence was experimental: Dasgupta et al. (2000) used photographs of nonfamous black and white people, with the assumption that they would be equally unfamiliar to subjects. The IAT using these photographs nevertheless showed a prowhite bias, and hence the authors argued that differential familiarity could not account for the finding.

We suggest, however, that this reasoning is mistaken. Specifically, the assumption that the black and white faces did not differ in familiarity because all of the photographs were of nonfamous people is not warranted. There is much evidence in the face recognition literature for the "other race effect," which refers to the finding that unknown/nonfamous faces belonging to other races are recognized more poorly relative to the own-race faces (e.g., Bothwell, Brigham, \& Malpass, 1989; Chiroro \& Valentine, 1995; O’Toole, Deffenbacher, Valentin, \& Abdi, 1994). There is a general consensus that this effect reflects the fact that faces belonging to one's own race are more familiar (see Tanaka, Kiefer, \& Bukach, 2004, for evidence that faces from a highly familiar racial group are recognized holistically). The role of familiarity is directly supported by Chiroro and Valentine's (1995) finding of a significantly reduced other-race effect in subjects who have high contact with other races. Thus, in the experiment by Dasgupta et al. (2000), given that the majority of subjects were nonblack (of the 75 subjects, 35 were Caucasians, 22 were Asians, and 18 were "other ethnicities," p. 320), they would have found the black faces less familiar than the white faces. Thus, the IAT effect observed with the face stimuli is entirely compatible with familiarity being the causal factor driving the IAT effect. (See also our discussion of the brain imaging and neuropsychological studies using face stimuli below.)

The second piece of evidence presented by Dasgupta et al. (2000) was statistical. First, the authors took a measure of familiarity of the names used in the IAT. Subjects were presented with the black and white names used as the target stimuli in the IAT randomly mixed with pseudonames, and the latency with which the real names were discriminated from pseudonames was assumed to index familiarity. This task showed that the white names were discriminated from pseudonames faster and more accurately than the black names $(711 \mathrm{msec}$ vs. $928 \mathrm{msec}$, and $3 \%$ vs. $18 \%$, respectively), suggesting that white 
names were indeed more familiar than black names. Dasgupta et al. (2000) then showed in a regression analysis that even when the white and black names were statistically equated on familiarity using this measure (i.e., regressing the familiarity advantage of white names against the size of the IAT effect and examining the point at which the familiarity advantage was zero), there was still a significant IAT effect (i.e., the $y$-intercept was positive). Dasgupta et al. (2000) took this finding to argue against the familiarity-based interpretation of the prowhite IAT effect.

This evidence, however, does not mean that familiarity did not play a role: On the contrary, the regression data showed a significant positive slope for the white-name familiarity advantage in the name IAT (i.e., as the familiarity advantage of the white names increased, so did the size of the name IAT effect). Moreover, equating the familiarity of individual stimuli does not rule out greater familiarity with whites as a category, as argued below.

Other studies claim to have ruled out familiarity by matching frequency of occurrence of individual exemplars in the contrasting categories-for example, Christian and Jewish surnames or black and white first names, based on the census data (e.g., Dasgupta, McGhee, Greenwald, \& Banaji, 1999, cited in Dasgupta et al., 2000; Rudman, Greenwald, Mellott, \& Schwartz, 1999, Experiment 1). In evaluating these studies, it is important to keep in mind how familiarity is assumed to modulate the IAT effect. Specifically, according to the salience asymmetry account and the stimulus-response compatibility account, the process of classification is facilitated by having a shared response to the figure categories. Thus, when asked to sort Jewish and Christian surnames into the two categories, Jewish subjects would focus on the "Jewish name" category as the figure category, and Christian subjects would focus on the "Christian name" category as the figure category, because it is more familiar. When there is a preexisting difference in relative familiarity of contrasting categories, as in this case, equating familiarity of individual stimuli used does not eliminate difference in familiarity of categories (just as using unknown faces does not eliminate the other-race effect in face recognition). Accordingly, the fact that the Jewish subjects showed a pro-Jewish IAT effect and the Christian subjects showed a pro-Christian IAT effect (Rudman et al., 1999, Experiment 1), when the individual Jewish and Christian surnames were matched on objective frequency (census) data does not rule out familiarity of the category as being responsible for driving the IAT effect. Similarly, other studies (e.g., Dasgupta et al., 1999, cited in Dasgupta et al., 2000) that equated familiarity of individual stimuli and found IAT effects do not rule out the familiarity-based account.

\section{Brain Imaging and \\ Neuropsychological Evidence}

Another type of evidence that has been used to argue for affect being the basis of the race IAT effect comes from an fMRI study that examined the relationship between the activation of the amygdala and the race IAT effect. On the basis of the assumption that the prowhite IAT effect reflects greater positive affect to white Americans, Phelps et al. (2000) hypothesized that the amygdala, which is generally assumed to be associated with emotional learning and evaluation, would be activated more when white participants viewed photographs of black faces than white faces, and that this differential amount of activation would be correlated with the IAT effect. Their Experiment 1 showed exactly this pattern when the stimuli consisted of photographs of unfamiliar white and black faces (taken from college yearbooks). Note, however, that correlation does not imply causality: The observed coupling between the race IAT effect and the amygdala activation does not mean that the greater negative affect toward black Americans was directly responsible for the IAT effect.

In fact, the results of Phelps et al.'s (2000) Experiment 2 are problematic for the affect-based interpretation. In that experiment, instead of the unfamiliar faces, photographs of positively rated famous black and white faces (e.g., of Michael Jordan, Bill Cosby, Tom Cruise, John F. Kennedy) were used. In contrast to their Experiment 1, the amygdala activity did not differ between the black and white faces, but the IAT still showed a prowhite bias. This finding indicates that the prowhite IAT effect can be dissociated from affect (as tapped by the amygdala activation). Similar decoupling between affect and the race IAT effect has also been reported in a recent neuropsychological study. Phelps, Cannistraci, and Cunningham (2003) tested patients with bilateral amygdala damage on the race IAT. On the assumption that damage to the amygdala impairs evaluations of affect, with unfamiliar black and white faces as stimuli (as in Experiment 1 of Phelps et al., 2000), the affect-based interpretation of the race IAT effect predicts little or reduced prowhite bias in these patients. On the contrary, these patients (all white Americans) showed prowhite IAT effect of the same size as the control subjects. These data indicating decoupling of the prowhite bias and amygdala activation are clearly incompatible with the view that affective valence is the causal factor driving the race IAT effect.

In contrast, the decoupling of the IAT effect and the amygdala activation is entirely compatible with the view that (as argued earlier) white subjects are more familiar with nonfamous white faces than with nonfamous black faces, and that this differential familiarity was responsible for the prowhite IAT effect. Furthermore, this interpretation explains the differential amygdala activation observed with the nonfamous white and black faces in Experiment 1 of Phelps et al. (2000): The suggestion is that the white faces were perceived as more attractive by the white subjects, because of greater familiarity. Rhodes et al. (2005) reported that Caucasian subjects rated ownrace composite male faces (computer-generated averaged composite faces) as more attractive than composite Japanese male faces. ${ }^{4}$ According to Rhodes et al. (2005), 
this own-race advantage would be expected on the basis of greater experience with own-race faces, the same factor that is responsible for an "average" face being perceived as attractive, as has been found consistently in the face recognition literature (see, e.g., Rhodes \& Zebrowitz, 2002). In Phelps et al.'s (2000, Experiment 1) study, then, if the white subjects perceived the own-race (white) faces as more attractive than the other-race (black) faces, this could have caused the differential amygdala activation observed. Note that this familiarity-mediated affect interpretation also explains the absence of differential amygdala activation in Experiment 2, because all the faces used in this experiment were famous and positively rated. In contrast, the view that white subjects find white faces more positive, irrespective of familiarity, could not explain this result.

Brain activation in regions other than the amygdala implicated in executive control have been investigated in white subjects' response to nonfamous black and white faces (Richeson et al., 2003). Richeson and colleagues found greater activity to black than to white faces in the right dorsolateral prefrontal cortex (DLPFC) and the right anterior cingulate cortex (ACC) and that the activity was correlated with the prowhite IAT effect. Insofar as these brain regions are not implicated in processing of affect, these findings are silent about the affectversus-familiarity debate and will not be discussed here. Familiarity-based interpretation of the above data and the additional data reported by Richeson et al. will be discussed later (see the Alternative Interpretations of the Race IAT Effects section).

In sum, activation in the brain regions assumed to be involved in processing of affect may be associated to, or decoupled from, the prowhite IAT effect. To the extent that familiarity can mediate affect, it seems more parsimonious to explain the relationship between the race IAT effect and the brain imaging and neuropsychological data in terms of familiarity rather than affect as the causal factor underlying the race IAT.

\section{Behavioral Evidence}

Another line of argument that has been used against familiarity as a determinant of the race IAT effect is to point to the fact that the IAT effect is predictive of behavioral measures of racial prejudice. McConnell and Leibold (2001) showed that the size of the prowhite IAT effect correlated with some behavioral measures of racial prejudice toward a black experimenter, such as the amount of smiling and speaking time. Similarly, Richeson and Shelton (2003) reported that the size of (white) subjects'prowhite IAT effect predicted measures of behavioral control (e.g., lack of body movement while answering, lack of hand movement) and measures of response modulation (e.g., apologizing for their response, long lead time in answering a question) during interaction with a black experimenter, but not with a white experimenter. These demonstrations have been used to argue for the criterion validity of the race IAT as a measure of racial prejudice.
It should be noted, however, that the observed correlations, while important, do not imply that the IAT taps affective valence rather than familiarity. People behave differently toward unfamiliar, unknown objects as opposed to familiar objects: The behavior toward unfamiliar objects is often characterized by avoidance. An example of this is food neophobia (many parents who try to introduce new food to their toddler offspring would be familiar with this experience). It is relevant to note here that several studies have demonstrated either no relation between food neophobia and liking for novel foods actually tasted (e.g., Pliner \& Hobden, 1992), or a weaker relation between neophobia and actual liking than between neophobia and expected liking (e.g., Arvola, Lahteenmaki, \& Tuorila, 1999). (The same parents above may also be familiar with saying to their toddler offspring "but how do you know you don't like it until you tried it!?") Thus it is an open question whether avoidance behavior is a reflection of negative affect, lack of familiarity, or both. Hence the correlation between the size of the race IAT effect and the behavioral measures toward the black-versus-white experimenter does not rule out the different degrees of familiarity with black and white Americans as the basis of the race IAT effect.

\section{Familiarity, Affect, and the Mere Exposure Effect}

We have put forward the case that familiarity, rather than affect, is the causal factor underlying the race IAT effect. Against this, a proponent of the affect-based interpretation of the race IAT could argue that the two interpretations are not functionally distinct by pointing to the mere exposure effect. This phenomenon refers to the finding that liking judgments are affected by prior exposure to stimuli: When asked to choose which stimulus they prefer, people choose the more familiar of the two. (For a very interesting analysis of the mere exposure effect with French impressionist paintings, see Cutting, 2003.) In the domain of social psychology, the mere exposure effect is typically taken as indicating that familiarity directly increases the affective value of stimuli (Zajonc, 1968, 2001). This view is based on the fact that liking judgments are increased even when prior exposure is subliminal and recognition judgments are at chance in a two-alternative forced choice paradigm (Kunst-Wilson \& Zajonc, 1980). If it is the case that familiarity directly increases liking, the view that familiarity is the factor underlying the race IAT effect does not differ in any real sense from the view that affect underlies the race IAT effect.

Against this, we point out that an alternative interpretation of the mere exposure effect suggests that familiarity does not directly increase the affective value of the stimulus, but instead, views the increase in liking judgments as a behavioral consequence of an attribution process. Mandler, Nakamura, and van Zandt (1987) demonstrated that the mere exposure effect is not limited to liking judgments but is also found when subjects judged the brightness, or darkness, of stimuli. They suggested that prior exposure increases fluency with which the 
stimulus is processed and that this enhanced fluency in turn influences the choice between two stimuli along any dimension, not just affect. Lee (2001) conceptually replicated this finding by showing that a single exposure of stimulus affected both density judgments and liking judgments in the same direction. Lee further showed that repeated exposure beyond a single exposure increased the liking judgment but not the density judgment, taking this to argue against the attribution interpretation and that increased familiarity directly enhances liking. However, Lee's finding is still interpretable in terms of an attribution process. Whittlesea and Price (2001) put forward the view that the misattribution of fluency occurs when subjects make judgments in a nonanalytic fashion, without scrutinizing the bases of their judgments. According to Whittlesea and Price, liking judgments are generally made nonanalytically and hence are more prone to misattribution of fluency than are other cognitive judgments. Within this view, Lee's finding is entirely consistent with the attribution interpretation if it is assumed that density judgments are made on more analytic bases than are liking judgments. In support of this interpretation, Whittlesea and Price found that when subjects were required to justify the bases of their liking judgments, such judgments were no more sensitive to the number of prior exposures than were recognition judgments; conversely, when subjects were instructed to make recognition judgments on a less analytic basis ("choose the stimulus which is more globally similar to a studied item"), recognition judgments were equally sensitive to the number of prior exposures.

In summary, the available evidence regarding the mere exposure effect does not conclusively indicate that familiarity directly increases affect. Accordingly, we contend that the view that the race IAT effect is based on familiarity is conceptually and functionally distinct from the view that the race IAT effect is based on affect.

\section{ALTERNATIVE INTERPRETATIONS OF THE RACE IAT EFFECTS}

We have argued that none of the studies reviewed presents definitive evidence that the race IAT effect taps affective valence, and that salience asymmetry based on differential familiarity with the race categories remains a viable mechanism. We will now reinterpret additional data that have been obtained with the race (or in-group vs. out-group) IAT in light of this.

\section{Implicit Partisanship Effect}

The implicit partisanship effect (Greenwald, Pickrell, $\&$ Farnham, 2002) refers to the finding that studying the names of members of a hypothetical group for just $45 \mathrm{sec}$ produced an apparent preference in an IAT (i.e., the studied names were found to be compatible with pleasant attributes) in comparison to a set of names that had not been studied (see also Ashburn-Nardo, Voils, \& Monteith, 2001, for a related finding). Greenwald, Pick- rell, and Farnham interpreted the effect as indicating that "there is an implicit evaluative benefit of category membership" (p. 378) and that "mere category membership carries attitudinal benefits" (p. 378). Alternatively, these findings may be interpreted in terms of salience asymmetry, on the basis of greater salience of studied names. Greenwald, Pickrell, and Farnham also considered this possibility, under the label of "mere categorization effect," but rejected it on the ground that their procedure included details that exceeded simple learning of a new category-specifically, the use of a fictitious scenario involving competition between teams and the request that subjects imagine that they lived in the same dormitory with members of the two teams. This argument is not convincing, however - there is no evidence that such a cover story was necessary to produce the effect. On the contrary, Greenwald, Pickrell, and Farnham's "Preliminary Experiment A" involved a control condition in which no such cover story was given, yet an IAT effect was found in favor of a set of names that was studied first. These results suggest that the apparent implicit partisanship effect may have little to do with group dynamics or evaluative aspect of attitudes, instead, reflecting greater salience of the "in-group" stimuli.

\section{Contextual Variations in the Race IAT}

Mitchell, Nosek, and Banaji (2003, Experiments 1 and 3 ) reported data that they interpreted as reflecting the malleability of attitudes. In their study, the IAT was used as an index of "automatic attitudes" toward the names of well-known black Americans and white Americans who were liked athletes (e.g., Michael Jordan) and disliked politicians, respectively. Their main finding was that depending on whether the classification was based on occupation ("athletes" vs. "politicians") or race ("black Americans" vs. "white Americans"), the apparent compatibility between the pleasant attribute and the same set of names was reversed. Specifically, with the occupationcategorization task, there was an apparent compatibility effect between black athletes with "good"; on the other hand, with the race-categorization task, there was an apparent compatibility effect between the same black athletes with "bad." This finding was interpreted by Mitchell et al. as reflecting that the automatic attitude toward an individual is flexible and depends on contextual cues. To use their example, "one may lament the fact that Charlton Heston is president of the National Rifle Association but admit that he was great in Planet of the Apes" (p. 458).

Although we find the Charlton Heston example a good indication of how one can hold ambivalent attitudes toward an individual, the experimental finding reported by Mitchell et al. (2003) may have little to do with such ambivalence. Their finding may be interpreted as showing the malleability of the IAT effect based on the relative salience of contrasting categories used. Specifically, it is suggested that whereas the category "white Americans" was more salient than the category "black Americans" and thus formed the figure category, the cat- 
egory "politicians" was less salient than "athletes" and hence was the background category. This could have come about for a number of reasons. In race classification, the white category may be more familiar than the black category for the usual reasons, or because in general there are more famous white individuals than famous black individuals. In contrast, in occupation classification, the features that define an athlete may be more salient than those that define a politician; alternatively, athletes as a category may be rated as more positive than politicians as a category, either in general, or as defined by the individual names used (a possibility consistent with the liking ratings of the individual names used). Any of these factors could have resulted in the reversal of the IAT effect. However, none of these interpretations say anything about evaluations of individuals, or how the context in which an individual is viewed changes the evaluation of the individual.

\section{Correlation Between the IAT Effect and the Effect of Interracial Contact on Executive Function}

Richeson et al. (2003; Richeson \& Shelton, 2003) reported that the size of the race IAT effect in white subjects was predictive of the impairment on a cognitive task following cross-race, but not same-race interaction. Subjects initially performed a race IAT using white and black names. They then had a brief interaction with either a black confederate or a white confederate, during which they were videotaped while being asked their opinion on two somewhat controversial topics - namely, the college's fraternity system and racial profiling in light of the September 11 attacks. Subsequently, they completed a Stroop color sorting task. The main finding was that the Stroop interference effect (the slower RT in the incongruent condition than in the congruent condition) was a linear function of the subjects' IAT effect when the Stroop task followed an interaction with a black confederate, but not when it followed an interaction with a white confederate. Richeson and Shelton took the results to suggest that cross-race interaction, "a particularly taxing exercise for highly prejudiced individuals, negatively affected performance on a subsequent test of executive function" (p. 287, italics ours). In addition, Richeson et al. reported brain imaging data that relate to the correlation between the prowhite IAT effect and the Stroop effect. Specifically, the prowhite IAT correlated with the amount of activity in the right ACC and DLPFC during viewing of black and white face stimuli; furthermore, the activity of the right DLPFC in response to black face stimuli statistically mediated the correlation between the size of the prowhite IAT effect and the Stroop interference effect when the Stroop task followed cross-race interaction, but not when it followed samerace interaction. The authors' own interpretation is that the activity of the DLPFC reflected inhibition of racial prejudice, on the basis that this region is known to be involved in inhibition of prepotent responses (e.g., Gehring \& Knight, 2000). In their words, "individuals with high scores on subtle measures of racial bias may put forth additional effort to control their thoughts and behaviors in order to live up to their egalitarian, nonprejudiced values" (p. 1326).

We are somewhat skeptical of this interpretation. By most accounts (for a review, see Kane \& Engle, 2002), the DLPFC is assumed to inhibit responses that are incompatible with task-relevant goals. In Richeson et al.'s (2003) study, during the imaging phase, subjects were required to decide whether a (black or white) face stimulus appeared on the right or left of the fixation. This task does not require face stimuli to be processed deeply, and hence even if a racist thought arose in response to the black face stimuli, it would be unlikely to interfere with the goal of deciding whether it was on the right or left of fixation. In other words, racist thoughts (and it is unclear whether they occurred) arising during passive viewing of photographs can hardly be described as a prepotent response under this task condition.

An alternative interpretation of the data suggests that the prowhite IAT effect indexed lack of familiarity with black Americans. According to this view, subjects with a large prowhite IAT effect would find the black faces particularly novel. The ACC is known to respond preferentially to novel stimuli even in a neutral behavioral context (e.g., Downar, Crawley, Mikulis, \& Davis, 2002), hence the greater activity of the ACC in response to black, relative to white, faces during passive viewing would be expected to correlate with the prowhite IAT effect. The activity of the DLPFC in response to the black face stimuli is also expected from the perspective that the ACC and the DLPFC form an attentional-control monitoring circuit. According to this view, the DLPFC is responsible for allocating attentional resources to novel stimuli, as evidenced by the finding that patients with damage to the DLPFC spend much less time looking at novel stimuli (Daffner et al., 2000). This would explain why the activity of the DLPFC was a statistical mediator of the correlation between the prowhite IAT effect and the Stroop interference effect following cross-race interaction: Subjects with a large prowhite IAT effect are those who are particularly unfamiliar with black Americans and hence would be adversely affected by the interaction. We believe this and other possibilities (see also Gehring, Karpinski, \& Hilton, 2003, for another interpretation) merit consideration before concluding that the DLPFC is responsible for inhibiting racist thoughts during passive viewing of photographs of black faces.

\section{CONCLUSION}

IAT effects have generally been interpreted in terms of conceptual association between the target and attribute dimensions - for example, reflecting positive evaluation of both the "flower" category and the "pleasant" attribute. However, in some IATs, there is no a priori association between the target and attribute dimensions. Consistent with a view suggested by Rothermund and Wentura (2004), we believe that the IAT effects in 
this second class are better interpreted in terms of salience asymmetry, based on dimensions like familiarity and linguistic markedness. In particular, we believe that the race IAT, perhaps one of the most studied forms of IAT, is a prime example of this second class of IAT. In contradiction to the claim that familiarity has been ruled out, our analysis of the studies using the race IAT suggested that these studies in fact did not provide evidence against familiarity. In light of this, we argued that alternative interpretations based on notions other than implicit racial prejudice should be considered for data based on the race IAT.

In closing, we believe that the fact that the race IAT effect reflects familiarity does not make it "vacuous" (Greenwald, Banaji, et al., 2002, p. 8, note 5). In particular, we believe that the correlation observed between behavior toward cross-race confederates and the race IAT effect (McConnell \& Liebold, 2001; Richeson et al., 2003; Richeson \& Shelton, 2003) has important implications for understanding how attitudes develop. Attitude comprises behavior, feelings, and evaluations, and there is a complex interplay between these aspects. Thus, the fact that familiarity influences behavior does not rule out the possibility that one's own behavior is interpreted, and evaluation is then constructed from it. We believe that such complexity befits a complex construct like attitude, and that studying the nature of interplay between the different aspects would be a worthy enterprise.

\section{REFERENCES}

Arvola, A., Lahteenmaki, L., \& Tuorila, H. (1999). Predicting the intent to purchase unfamiliar and familiar cheeses: The effects of attitudes, expected liking, and food neophobia. Appetite, 32, 113-126.

Ashburn-NARdo, L., Voils, C. I., \& MonteIth, M. J. (2001). Implicit associations as the seeds of intergroup bias: How easily do they take root? Journal of Personality \& Social Psychology, 81, 789-799.

Bothwell, R. K., Brigham, J. C., \& Malpass, R. S. (1989). Cross-racial identification. Personality \& Social Psychology Bulletin, 15, 19-25.

Brendl, C. M., Markman, A. B., \& Messner, C. (2001). How do indirect measures of evaluation work? Evaluating the inference of prejudice in the Implicit Association Test. Journal of Personality \& Social Psychology, 81, 760-773.

Chiroro, P., \& Valentine, T. (1995). An investigation of the contact hypothesis of the own-race bias in face recognition. Quarterly Journal of Experimental Psychology, 48A, 879-894.

Chо, Y. S., \& Proctor, R. W. (2003). Stimulus and response representation underlying orthogonal stimulus-response compatibility effects. Psychonomic Bulletin \& Review, 10, 45-73.

CLARK, E. V. (1973). What's in a word? On the child's acquisition of semantics in his first language. In T. E. Moore (Ed.), Cognitive development and the acquisition of language (pp. 65-110). New York: Academic Press.

Clark, H. H. (1973). Space, time, semantics, and the child. In T.E. Moore (Ed.), Cognitive development and the acquisition of language (pp. 2763). New York: Academic Press.

Cunningham, W. A., Preacher, K. J., \& Banaji, M. R. (2001). Implicit attitude measures: Consistency, stability, and convergent validity. Psychological Science, 12, 163-170.

CutTing, J. E. (2003). Gustave Caillebotte, French Impressionism, and mere exposure. Psychonomic Bulletin \& Review, 10, 319-343.

Daffner, K. R., Mesulam, M. M., Holcomb, P. J., Calvo, V., Acar, D., Chabrerie, A., Kikinis, R., Jolesz, F. A., Rentz, D. M., \& Scinto, L. F. (2000). Disruption of attention to novel events after frontal in- jury in humans. Journal of Neurology, Neurosurgery, \& Psychiatry, 68, 18-24.

Dasgupta, N., Greenwald, A. G., \& Banaji, M. R. (2003). The first ontological challenge to the IAT: Attitude or mere familiarity? Psychological Inquiry, 14, 238-243.

Dasgupta, N., McGhee, D. E., Greenwald, A. G., \& Banaji, M. R. (2000). Automatic preference for white Americans: Eliminating the familiarity explanation. Journal of Experimental Social Psychology, 36, 316-328.

De Houwer, J. (2001). A structural and process analysis of the Implicit Association Test. Journal of Experimental Social Psychology, 37, 443-451.

Downar, J., Crawley, A. P., Mikulis, D. J., \& Davis, K. D. (2002). A cortical network sensitive to stimulus salience in a neutral behavioral context across multiple sensory modalities. Journal of Neurophysiology, 87, 615-620.

FAZIO, R., \& OLSON, M. A. (2003). Implicit measures in social cognition research: Their meaning and use. Annual Review of Psychology, 54, 297-327.

Gehring, W. J., Karpinski, A., \& Hilton, J. L. (2003). Thinking about interracial interactions. Nature Neuroscience, 6, 1241-1244.

Gehring, W. J., \& Knight, R.T. (2000). Prefrontal-cingulate interactions in action monitoring. Nature Neuroscience, 3, 516-520.

Gray, N. S., MacCulloch, M. J., Smith, J., Morris, M., \& SnowDEN, R. J. (2003). Violence viewed by psychopathic murderers: Adapting a revealiing test may expose those psychopaths who are most likely to kill. Nature, 423, 497-498.

Greenwald, A. G., \& BanaJI, M. R. (1995). Implicit social cognition: Attitudes, self-esteem, and stereotypes. Psychological Review, 102, 4-27.

Greenwald, A. G., Banaji, M. R., Rudman, L. A., Farnham, S. D., Nosek, B. A., \& Mellott, D. S. (2002). A unified theory of implicit attitudes, stereotypes, self-esteem, and self-concept. Psychological Review, 109, 3-25.

Greenwald, A. G., McGhee, D. E., \& Schwartz, J. L. K. (1998). Measuring individual differences in implicit cognition: The implicit association test. Journal of Personality \& Social Psychology, 74, 1464-1480.

Greenwald, A. G., Nosek, B. A., Banaji, M. R., \& Klauer, K. C. (2004). Validity of the salience asymmetry interpretation of the IAT: Comment on Rothermund and Wentura (2004). Journal of Experimental Psychology: General (in press).

Greenwald, A. G., Pickrell, F. E., \& Farnham, S. D. (2002). Implicit partisanship: Taking sides for no reason. Journal of Personality \& Social Psychology, 83, 367-379.

Hansen, C. H., \& Hansen, R. D. (1988). Finding the face in the crowd: An anger superiority effect. Journal of Personality \& Social Psychology, 54, 917-924.

JACOBY, L. L. (1996). Dissociating automatic and consciously controlled effects of study/test compatibility. Journal of Memory \& Language, 35, 32-52.

Kane, M. J., \& Engle, R. W. (2002). The role of prefrontal cortex in working-memory capacity, executive attention, and general fluid intelligence: An individual-differences perspective. Psychonomic Bulletin \& Review, 9, 637-671.

KAne, M. J., \& Engle, R. W. (2003). Working-memory capacity and the control of attention: The contributions of goal neglect, response competition, and task set to Stroop interference. Journal of Experimental Psychology: General, 132, 47-70.

KIM, D.-Y. (2003). Voluntary controllability of the Implicit Association Test (IAT). Social Psychology Quarterly, 66, 83-96.

Klauer, K. C., \& Mierke, J. (2004). Task-set inertia, attitude accessibility, and compatibility-order effects: New evidence for a task-set switching account for the IAT effect. Personality \& Social Psychology Bulletin (in press).

KunST-Wilson, W. R., \& ZAJONC, R. B. (1980). Affective discrimination of stimuli that cannot be recognized. Science, 207, 557-558.

LEE, A. Y. (2001). The mere exposure effect: An uncertainty reduction explanation revisited. Personality \& Social Psychology Bulletin, 27, 1255-1266.

LyONS, J. (1977). Semantics I. London: Cambridge University Press. 
Mandler, G., Nakamura, Y., \& van Zandt, B. J. S. (1987). Nonspecific effects of exposure on stimuli that cannot be recognized. Journal of Experimental Psychology: Learning, Memory, \& Cognition, 13, 646-648.

McConnell, A. R., \& Leibold, J. M. (2001). Relations among the Implicit Association Test, discriminatory behavior, and explicit measures of racial attitudes. Journal of Experimental Social Psychology, 37, 435-442.

McFarland, S. G., \& Crouch, Z. (2002). A cognitive skill confound on the Implicit Association Test. Social Cognition, 20, 483-510.

Meiran, N. (2000). Modelling cognitive control in task-switching. Psychological Research, 63, 234-249.

Mierke, J., \& KlaUer, K. C. (2001). Implicit association measurement with the IAT: Evidence for effects of executive control processes. Zeitschrift fur Experimentelle Psychologie, 48, 107-122.

Mitchell, J. P., Nosek, B. A., \& BanajI, M. R. (2003). Contextual variations in implicit evaluation. Journal of Experimental Psychology: General, 132, 455-469.

Monteith, M. J., Voils, C. I., \& Ashburn-Nardo, L. (2001). Taking a look underground: Detecting, interpreting, and reacting to implicit racial biases. Social Cognition, 19, 395-417.

Nosek, B. A., Banaji, M., \& Greenwald, A. G. (2002). Harvesting implicit group attitudes and beliefs from a demonstration web site. Group Dynamics: Theory, Research, \& Practice, 6, 101-115.

Ohman, A., Lundqvist, D., \& Esteves, F. (2001). The face in the crowd revisited: A threat advantage with schematic stimuli. Journal of Personality \& Social Psychology, 80, 381-396.

OlsON, M. A., \& FAZIO, R. H. (2003). Relations between implicit measures of prejudice: What are we measuring? Psychological Science, 14, 636-639.

O’Toole, A. J., Deffenbacher, K. A., Valentin, D., \& Abdi, H. (1994). Structural aspects of face recognition and the other-race effect. Memory \& Cognition, 22, 208-224.

Phelps, E. A., Cannistraci, C. J., \& Cunningham, W. A. (2003). Intact performance on an indirect measure of race bias following amygdala damage. Neuropsychologia, 41, 203-208.

Phelps, E. A., O’Connor, K. J., Cunningham, W. A., Funayama, E. S., Gatenby, J. C., Gore, J. C., \& Banaji, M. R. (2000). Performance on indirect measures of race evaluation predicts amygdala activation. Journal of Cognitive Neuroscience, 12, 729-738.

Pliner, P., \& Hobden, K. (1992). Development of a scale to measure the trait of food neophobia in humans. Appetite, 19, 105-120.

Rhodes, G., Lee, K., Palermo, R., Weiss, M., Yoshikawa, S., Clissa, P., Williams, T., Peters, M., Winkler, C., \& JefFrey, L. (2005). Attractiveness of own-race, other-race, and mixed-race faces. Perception, 34, 319-340.

Rhodes, G., \& Zebrowitz, L. A. (Eds.) (2002). Facial attractiveness: Evolutionary, cognitive, and social perspectives. Westport, CT: Ablex.

Richeson, J. A., Baird, A. A., Gordon, H. I., Heatherton, T. F., Wyland, C. L., Trawalter, S., \& Shelton, J. N. (2003). An f MRI investigation of the impact of interracial contact on executive function. Nature Neuroscience, 6, 1323-1328.

Richeson, J. A., \& Shelton, J. N. (2003). When prejudice does not pay: Effects of interracial contact on executive function. Psychological Science, 14, 287-290.

Rogers, R. D., \& Monsell, S. (1995). Costs of predictable switch between simple cognitive tasks. Journal of Experimental Psychology: General, 124, 207-231.

Rothermund, K., \& Wentura, D. (2001). Figur-Grund Asymmetrien im Implicit Association Test (IAT). Zeitschrift fur Experimentelle Psychologie, 48, 94-106.

Rothermund, K., \& Wentura, D. (2004). Underlying processes in the Implicit Association Test (IAT): Dissociating effects of salience and valence. Journal of Experimental Psychology: General, 133, 139-165.

Rudman, L. A., Greenwald, A. G., Mellott, D. S., \& Schwartz, J. L. K. (1999). Measuring the automatic components of prejudice: Flexibility and generality of the Implicit Association Test. Social Cognition, 17, 437-465.

Steffens, M. C., \& Plewe, I. (2001). Items' cross-category associations as a confounding factor in the Implicit Association Test. Zeitschrift fur Experimentelle Psychologie, 48, 123-134.

STRAYER, D. L., \& JOHNSTON, W. A. (2000). Novel popout is an attention- based phenomenon: An ERP analysis. Perception \& Psychophysics, 62, 459-470.

TanaKa, J. W., Kiefer, M., \& Bukach, C. M. (2004). A holistic account of the own-race effect in face recognition: Evidence from a crosscultural study. Cognition, 93, B1-B9.

Teachman, B. A., GregG, A. P., \& Woody, S. R. (2001). Implicit associations for fear-relevant stimuli among individuals with snake and spider fears. Journal of Abnormal Psychology, 110, 226-235.

TipPles, J., Young, A. W., Quinlan, P., Broks, P., \& Ellis, A. W. (2002). Searching for threat. Quarterly Journal of Experimental Psychology, 55A, 1007-1026.

Wang, Q., CaVanagh, P., \& Green, M. (1994). Familiarity and pop-out in visual search. Perception \& Psychophysics, 56, 495-500.

Whittlesea, B. W. A., \& Price, J. R. (2001). Implicit/explicit memory versus analytic/nonanalytic processing: Rethinking the mere exposure effect. Memory \& Cognition, 29, 234-246.

Wilson, T. D., LindSEY, S., \& Schooler, T. Y. (2000). A model of dual attitudes. Psychological Review, 107, 101-126.

ZaJonc, R. B. (1968). Attitudinal effects of mere exposure. Journal of Personality \& Social Psychology, 9(2, Pt. 2), 1-27.

ZAJONC, R. B. (2001). Mere exposure: A gateway to the subliminal. Current Directions in Psychological Science, 10, 224-228.

Zebrowitz, L. A., Montepare, J. M., \& LeE, H. K. (1993). They don't all look alike: Individual impressions of other racial groups. Journal of Personality \& Social Psychology, 65, 85-101.

Zebrowitz, L. A., \& RHodes, G. (2002). Nature let a hundred flowers bloom: The multiple ways and wherefores of attractiveness. In G. Rhodes \& L.A. Zebrowitz (Eds.), Facial attractiveness: Evolutionary, cognitive, and social perspectives (pp. 262-293). Westport, CT: Ablex.

\section{NOTES}

1. In particular, due to space constraints, we are unable to discuss the cognitive skills account proposed by McFarland and Crouch (2002) and its implication for the role of executive function in the IAT.

2. In particular, Mierke and Klauer (2001) pointed out a logical flaw with the spreading activation account. Specifically, the compatible and incompatible conditions in the IAT do not differ with respect to stimulus composition, but only in the assignment of responses to the contrasting categories. Accordingly, the priming process would be the same in the compatible and incompatible conditions, and hence the spreading activation mechanism cannot explain the IAT effect.

3. In a recent commentary on the salience asymmetry account by the originators of the IAT (Greenwald, Nosek, Banaji, \& Klauer, 2004), they stated that unlike Rothermund and Wentura (2004), who assumed association to mean semantic/conceptual association based on the meaning of the stimuli comprising the target and attribute categories, Greenwald et al. (2004) took a "theory-uncommitted conception of association," without committing themselves to "any theory of the structure of associative mental representation." We find this atheoretical conception of association troublesome, because it confounds explanation (mechanism) and data. Specifically, in the absence of a commitment to a theory of association, association can be defined only post hoc, by the data. Thus, the target and attribute dimensions are said to be associated when there is an IAT effect, and unassociated when there is not. The observed IAT effect is then explained in terms of an "atheoretical" association. This is clearly circular, and the claim that the IAT effect taps association between the target and attribute dimensions can never be falsified.

4. We should point out that the own-race faces are not always rated as more attractive than the other-race faces (e.g., Zebrowitz, Montepare, $\&$ Lee, 1993). This is explained by the fact that the attractiveness rating is influenced by many factors (Zebrowitz \& Rhodes, 2002), including perceived health, averageness, symmetry, pleasantness of expression, youthfulness, and sexual dimorphism, with familiarity being just one of them. Thus, whether an effect of familiarity would be observed would depend on what factors are salient in the set of stimulus faces used.

(Manuscript received June 23, 2004; revision accepted for publication February 4, 2005.) 